\title{
The effect of head position on size discrimination
}

PETER W. ZINKUS and PAUL T. MOUNTJOY. Western Michigan University, Kalamazoo, Mich. 49001

Twenty Ss were required to match the size and distance of equal-size disks in different planes of space. Head position was shown to be significantly related to the $S^{\prime}$ inability to make these discriminations. Evidence was also given to support the hypothesis of a vestibular contribution to space perception.

Changed head position, and the resulting stimulation of the vestibular apparatus, has recently been studied in its relationship with the visual processes. Research by Thor $\&$ Wood (1966) indicated that when an overhead target was viewed by Ss, it was judged to be smaller and farther away than a target of the same size and distance on the horizontal plane. In accounting for their results, they formulated a vestibular hypothesis which related changed head position to the resulting visual distortions. They also cited research by Grusser, Chornels, and Saur, which indicated that visual input is indeed modified by vestibular stimulation at the cortical level.

In another study, Wood, Zinkus, \& Mountjoy (1968) allowed Ss to manipulate the distance of equal-size targets, placed in different planes of space, in a totally darkened room. They found that Ss consistently placed the overhead target closer than the horizontal and again judged the overhead disk as smaller and farther away. Similar results were also obtained by Van Eyl (1968) who allowed Ss to manipulate the distance of equal-size disks in different planes of space.

The important aspect of the above-mentioned studies, however, is that support for a visual-vestibular interaction hypothesis was added to a growing body of research of aerospace medicine. Baker (1965) reviews many of these studies which indicate the effects of vestibular stimulation on visual acuity, brightness discrimination, and visual illusions. METHOD

The research reported in this paper was designed to more carefully isolate the effects of changed head position, and the resulting vestibular stimulation, on the visual capabilities of the human organism. Ss were seated in a spring-loaded office chair, which was fitted with a head rest and bolted securely to the floor. The room was totally darkened and the chair was enclosed on the sides and top to eliminate all extrinsic light sources. Three apertures, one in front of the chair, one $90 \mathrm{deg}$ to the right, and one in the top, allowed viewing of the stimulus disks. The chair was fitted with a mercury switch which would allow illumination of the front disk when the chair was in the upright position, but would terminate it when the chair was tilted back. Thus, a $S$ could not view two of the disks concurrently.

The disks were $3 \mathrm{in}$. in diam and illuminated by very low-intensity bulbs. Also, red filters were used so that the disks did not illuminate parts of the room.

Twenty Ss were individually blindfolded and led into the experimental room where they were seated in the chair. When the blindfold was removed, the $S$ could view the front disk through the aperture and then, by tilting back in the chair, view the overhead disk. He could not, however, view both disks simultaneously. The front disk was placed at a standard distance from the S's eyes and he was then instructed to tilt back in the chair and place the overhead disk the same distance from his eyes. The $S$ instructed the $E$ to raise or lower the overhead disk and could view each disk as many times as he desired in order to make a precise judgment. Four trials were conducted with each $S$ in this manner, with the front disk placed at a distance of $50,60,70$, and 80 in. from his eyes.
The second phase of the study required the $S$ to view the front disk and then swivel the chair $90 \mathrm{deg}$ to the right and match the distance of an identical disk with the one in the front. Again, the $S$ could not view both disks simultaneously, but had to view one, then swivel the chair to view the other. Four trials were conducted in this phase with the front standard placed at distances of $40,50,60$, and $70 \mathrm{in}$. from the S's eyes.

Half of the Ss began with Phase 1 while the other half began with Phase 2. After the first trial, Ss were asked by the E what kind of cues they were using in making their judgments. All Ss but one indicated that they were matching sizes.

\section{RESULTS AND DISCUSSION}

The results of this study seem to indicate that changed head position, relative to the direction of the force of gravity, was the critical factor in producing the gross distortions in size-distance discrimination when the objects are viewed in different planes of space. In Phase 1 all 20 Ss placed the overhead target considerably closer to their eyes than the front disk. The average of the mean discrepancy for four trials with each $S$ showed that the overhead disk was placed 17.80 in. closer than the front with a range from 14.25 to $25.00 \mathrm{in}$. In Phase 2, however, Ss averaged 1.38 in. of mean discrepancy for four trials with a range from $+2.50 \mathrm{in}$. to $-2.00 \mathrm{in}$. On some trials, Ss overestimated the side disk by a small amount and in others they underestimated.

A test of significance of the difference between the means of Phase 1 and Phase 2, using a " $t$ " for matched pairs, indicated that they differed beyond the .001 level of significance $(t=23.657)$.

The important consideration seems to be the effect of changed head position, relative to the direction of the force of gravity, on a S's ability to discriminate size. In Phase 1, with the disks located on the horizontal and vertical planes, Ss grossly overestimated the size of the front disk or, conversely, underestimated the overhead target. Ss indicated to the $E$ that they were matching sizes and that the overhead disk appeared smaller and farther away when actually equidistant with the front disk. They seemed to compensate for this effect by placing the overhead target closer to a significant degree. In Phase 2, however; head tilt was not involved and Ss were quite accurate in matching the disks. Since the starting phase was alternated for each $\mathbf{S}$, proficiency on either phase could not be attributed to a practice effect. Also, the time intervals between viewing the front disk and tilting or swivelling to view the other disks in each phase were controlled by the $E$ in order that they would be equal. In this way, proficiency could not be the result of memory factors due to a shorter interval on either phase.

The results of this study are compatible with a great deal of research, both physiological and behavioral, on the effects of vestibular stimulation on visual processes. The fact that vestibular stimulation can be accomplished by head tilt and that this system has been found to interact significantly, at the cortical level, with visual processes, does seem to add strength to a visual-vestibular interaction hypothesis.

\section{REFERENCES}

BAKER, G. A. Visual capabilities in the space environment. London: Pergamon Press, 1965.

THOR, D. H., \& WOOD, R. J. The moon illusion. Paper presented at American Psychological Association, September, 1966.

VAN EYL, F. P. The moon illusion. Paper presented at Michigan Academy of Arts, Science, and Letters, March, 1968.

WOOD, R. J., ZINKUS, P. W., \& MOUNTJOY, P. T. The vestibular hypothesis of the moon illusion. Psychonomic Science, 1968, $11,356$. 